\title{
Oral fluid matrix metalloproteinase (MMP)-8 as a diagnostic tool in chronic periodontitis
}

\author{
This article was published in the following Dove Press journal: \\ Metalloproteinases In Medicine \\ 27 May 2016 \\ Number of times this article has been viewed
}

\author{
Patricia Hernández-Ríos' \\ Marcela Hernández ${ }^{2,3}$ \\ Mauricio Garrido' \\ Taina Tervahartiala ${ }^{4}$ \\ Jussi M Leppilahti ${ }^{4}$ \\ Heidi Kuula ${ }^{4}$ \\ Anna Maria Heikkinen ${ }^{4}$ \\ Päivi Mäntylä ${ }^{4}$ \\ Nilminie Rathnayake ${ }^{5}$ \\ Solomon Nwhator ${ }^{6}$ \\ Timo Sorsa ${ }^{4,5}$ \\ 'Department of Conservative \\ Dentistry, ${ }^{2}$ Department of Pathology \\ and Oral Medicine, ${ }^{3}$ Laboratory of \\ Periodontal Biology, Department \\ of Conservative Dentistry, Faculty \\ of Dentistry, University of Chile, \\ Santiago, Chile; ${ }^{4}$ Department of Oral \\ and Maxillofacial Diseases, Institute \\ of Dentistry, Helsinki University \\ and Helsinki University Central \\ Hospital, Helsinki, Finland; ${ }^{5}$ Division \\ of Periodontology, Department of \\ Dental Medicine, Karolinska Institutet, \\ Stockholm, Sweden; ${ }^{6}$ Department of \\ Preventive and Community Dentistry, \\ Faculty of Dentistry, College of \\ Health Sciences, Obafemi Awolowo \\ University, Ife, Nigeria
}

Correspondence:Timo Sorsa Department of Oral and Maxillofacial Diseases, Institute of Dentistry, Helsinki University and Helsinki University Central Hospital, PO Box 63 (Haartmaninkatu 8), $\mathrm{Fl}-000 \mathrm{I} 4$ University of Helsinki, Helsinki, Finland

Tel +358294 I91।

Email timo.sorsa@helsinki.fi submit your manuscript | www.dovepress.com

\begin{abstract}
Periodontal diseases that affect the marginal and apical periodontium result from the interaction between bacterial biofilm and the host response. Oral fluid biomarkers might aid clinical diagnosis. Matrix metalloproteinases (MMPs) are a family of 24 proteases that act in physiological and pathological conditions. They can degrade almost all extracellular matrix constituents and regulate inflammatory processes. They are mainly inhibited by tissue inhibitors of metalloproteinases. The aim of this study was to perform a current literature review with a special reference on the diagnostic and clinical utility of oral fluid MMPs, especially MMP-8, and their inhibitors in periodontal and oral diseases. MMP-8 is the main collagenolytic MMP detected in oral fluids, such as saliva, oral mouthrinse, gingival crevicular fluid, and peri-implant fluid. MMP-8, and potentially MMP-9, in oral fluids represent strong biomarker candidates associated especially with periodontal disease diagnosis, severity, progression, and follow-up. Additionally, they show diagnostic potential for systemic conditions, such as pregnancy, myocardial infarction, and smoking. A commercially available mouthrinse, active MMP-8 chair-side/ point-of-care lateral flow immunoassay, shows enough sensitivity and specificity to detect clinical signs of periodontitis. The current literature supports that high MMP-8 levels reflect the loss of periodontal supporting tissues rather than inflammation, representing a potentially useful sidediagnostic point-of-care oral disease biomarker, especially in periodontal diseases.
\end{abstract}

Keywords: gingival crevicular fluid, saliva, biomarkers

\section{Introduction}

Periodontal diseases are chronic infections that affect the gingiva (gingivitis) and the supporting tissues of the teeth, namely, the alveolar bone, periodontal ligament, and radicular cementum in periodontitis. ${ }^{1-4}$ The later can either affect the marginal periodontium (ie, chronic periodontitis) or the apical periradicular tissues, known as apical periodontitis. ${ }^{5}$ They can be considered a global health problem that can lead to tooth loss, widespread oral health dysfunction, and increased susceptibility to other systemic diseases, such as cardiovascular diseases and stroke, diabetes, preterm delivery of low-birth-weight babies, arthritis, and lung inflammation. ${ }^{6-8}$

Periodontitis results from the interaction between bacterial biofilms and the host immune response. Because of its high prevalence and potential systemic health impact, periodontal diseases must be properly detected and treated, in order to preserve, improve, and maintain the remaining teeth, periodontium, or peri-implant tissues in health, comfort, esthetics, and function. ${ }^{9}$

Periodontal diagnosis is presently based on the measurement of a set of clinical parameters that includes probing depth (PD), clinical attachment level (CAL), bleeding 
on probing, plaque index, and radiographic recordings. ${ }^{4,6}$ However, in large-scale studies, these measurements are laborious, expensive, and time-consuming and require trained dental professionals. ${ }^{10}$ Besides, they display information about the past instead of current periodontal tissue breakdown, with an inability to predict future destruction, disease progression, or the patient's response to treatment. ${ }^{6,11,12}$ Some limitations of the diagnostic methods are basically derived from the episodic and multifactorial characteristics of periodontal diseases, which are further complicated by the facts that different teeth, and even tooth sites within the same patient, are affected by varying degrees of disease severity and may or may not undergo progression. ${ }^{13,14}$ Because the loss of periodontal supporting tissues is cumulative and irreversible, complementary methods aiding early diagnosis and preventing progression are highly desirable.

There is a current need to find a reliable complementary diagnostic tool that ideally aids to screen, differentiate site activity, predict future tissue destruction, and monitor the response to the therapy and medication of periodontal diseases. ${ }^{6}$ Oral fluids, such as gingival crevicular fluid (GCF), saliva, mouthrinse, and peri-implant sulcular fluid (PISF) are easily obtained by noninvasive methods and contain various molecular host immune and bacterial-derived components, often referred to as biomarkers. Oral fluid biomarkers can reflect several oral physiological and pathological conditions, including marginal periodontitis, apical periodontitis, periimplantitis, and orthodontic tooth movement, among others. Qualitative and quantitative changes in oral fluid biomarkers have demonstrated to exert diagnostic and prognostic potential, especially in chronic periodontitis. ${ }^{5,6,15}$ Saliva and mouth-rinse represent pooled samples at the patient-level, while GCF and PISF provide site-specific analysis.

Among host-derived biomarker candidates, matrix metalloproteinases (MMPs) represent a large family of calcium-dependent, zinc-containing proteases that act in physiological and pathological conditions. ${ }^{16-18}$ Currently, there are 24 genetically distinct, but structurally related, human MMPs types, which are divided into subgroups, such as collagenases (MMP-1, -8, and -13), gelatinases (MMP-9 and -2), membrane-type MMPs (MMP-14, -15, -16, -17, -24, and -25$)$, and others. ${ }^{19}$ Together, they can degrade almost all extracellular matrix and basement membrane constituents. They can also regulate cellular and inflammatory processes, through limited and decisive proteolysis of nonmatrix bioactive substrates (enzymes, chemokines, cytokines, growth factors, complement components, receptors, and serum components, among others)..$^{4,20-22}$ Downregulation of MMP-8 to physiological or normal levels appears to contribute to the resolution of inflammation through the processing of anti-inflammatory chemokines and cytokines and suggest, at least in part, also a defensive role for MMP-8 in periodontitis..$^{23,24}$

The activities of MMPs can be regulated by delicate inductive cascades through their gene expression, the conversion of zymogen to the active enzyme, and the presence of specific inhibitors. ${ }^{25}$ Tissue inhibitors of metalloproteinases (TIMPs)-1, $-2,-3$, and -4 represent the main MMP physiological inhibitors, ${ }^{19}$ and both, inhibitors and activating factors (MMPs and reactive oxygen species as other host and microbial proteases) determine the activities of MMPs in periodontitis. Interactions between their inhibitors and activating factors will thus determine MMP activity, which finally accounts for the rate of matrix turnover or destruction, ${ }^{6,26}$ as well as immune bioactive substrate processings. Total MMP levels can be measured by enzyme-linked immunosorbent and immunofluoromentric assays (ELISA and IFMA, respectively). The variety of MMP isoforms, proforms, active forms, fragmented species, and others, can be analyzed by Western immunoblotting, while the intrinsic and total MMP activities can be assessed by functional MMP activity assays, such as zymography or commercially available catalytic and immune assays.

Collagen I represents the bulk component of the periodontal extracellular matrix. Accordingly, collagenase or collagenolytic MMPs (MMP-1, -8, -13, and -14) and gelatinase MMPs (MMP-2 and -9) play a pivotal role in the loss of periodontal support on the basis of their collagen-degrading properties. ${ }^{27}$ Collagenases process native collagen I and III, and gelatinases further degrade the resultant denatured collagens. ${ }^{28}$ Among them, the main MMPs involved in periodontal tissue destruction are MMP-8, -13, and -9.,29

MMP- 8 constitutes the main collagenolytic MMP detected in gingival tissue and oral fluids ( $80 \%$ of collagenases in GCF) and has emerged as a promising periodontal biomarker. ${ }^{30}$ The major cellular source of MMP-8 are polimorphonuclear neutrophils (PMN), but it can also be produced by various non-PMN-lineage cells such as gingival fibroblasts, endothelial cells, epithelial cells, plasma cells, macrophages, and bone cells. ${ }^{31} \mathrm{MMP}-8$ has been related to periodontal disease diagnosis, severity of periodontal inflammation, progression, and treatment follow-up. ${ }^{32}$

MMP-13 has been observed in gingival sulcular epithelium, fibroblasts, macrophages, plasma cells, and osteoblasts; and MMP-13, together with MMP-9, has been associated with bone resorption. ${ }^{33-37}$ MMP-9 is also present in PMN granules, 
as a variety of other periodontal cell types, and it represents the main gelatinase in oral fluids. MMP-9 levels and active forms have been directly associated with periodontal inflammation and breakdown. ${ }^{38}$ Other MMPs that are also detected in oral fluids (GCF, PISF, saliva, and mouthrinse) in minor quantities are MMP-1, $-2,-3,-7,-12,-14,-25$, and -26 and TIMP-1 and $-2 .^{6,27}$ The aim of this study is to perform a current literature review with a special reference on the diagnostic and clinical utility of oral fluid MMPs, especially MMP-8, and their inhibitors in periodontal and oral diseases.

\section{MMPs as diagnostic biomarkers of periodontal diseases in saliva and mouthrinse}

Saliva is a clear, slightly acidic oral secretion, mainly produced by the major (parotid, submandibular, and sublingual) and minor salivary glands. ${ }^{39}$ It is composed of organic and inorganic compounds, with bacterial and host proteins, as well as dietary, serum, and blood components, leaked to the mouth by GCF or oral wounds. ${ }^{29,40}$

Saliva as a diagnostic fluid has a long history and constitutes a noninvasive, readily available, easily collected sample or matrix. ${ }^{6,41}$ Saliva collection methods through drooling, the utilization of cotton swabs, or paraffin gum chewing show a similar protein composition, with small differences in saliva volume. ${ }^{10,29}$ It can reflect the aspects of oral and systemic health status, aiding in diagnostics of cancers, cardiovascular diseases, immunologic syndromes, and hereditary deficiencies. ${ }^{29,42,43}$ Additionally, other environmental and systemic factors, such as smoking, diabetes, and medications, may eventually also influence biomarker concentrations. ${ }^{44-46}$ In periodontal disease, saliva constitutes an overall view of the disease status, with a pooled sample constituted by all periodontal sites and saliva secretion. ${ }^{47}$

MMP-8 has been described as one of the strongest salivary biomarkers for detecting alveolar bone destruction, associated with different clinical and radiological parameters, such as deepened periodontal pockets, progression of attachment loss, alveolar bone loss, and bleeding on probing (BOP). ${ }^{10}$ MMP-8 (mainly detected by IFMA), TIMP-1, MMP-8/TIMP-1 molar ratio, and carboxy-terminal telopeptide of type I collagen (ICTP) are able to distinguish between periodontitis and control groups, defined by bone resorption or the presence of periodontal pockets and ongoing periodontal attachment loss. ${ }^{46,48}$

MMP-8 has been observed at higher concentrations in generalized severe periodontal bone destruction than localized alveolar resorption, together with the increase of the MMP-8/TIMP-1 molar ratio and interleukin (IL)-1 $\beta$, a proinflammatory cytokine. ${ }^{46}$ MMP-9 and MMP-13 have been linked to MMP-8 and higher concentrations associated with generalized periodontitis, even when the highest levels of MMP-9 and -13 are found in localized periodontitis patients. ${ }^{49}$ Indeed, elevated levels of MMP-8 and -9 are found in advanced periodontitis patients in comparison with a healthy/gingivitis group of subjects, and these biomarkers, in combination with red complex periodontal pathogens (such as Porphyromonas gingivalis and Treponema denticola), can more accurately predict the different disease categories. ${ }^{50}$ MMP-14 measurements in other studies, do not show any difference between generalized periodontitis, localized periodontitis, and control group. ${ }^{48}$ Salivary MMP- 8 has also been associated with the response to therapy and medications. ${ }^{26,51,52}$ MMP-8 and IL-1 $\beta$ decrease significantly in relation to scaling and root planning, and MMP-8 shows significant correlations with the changes in posttreatment clinical parameters. ${ }^{52}$

It should be considered that the diagnostic value of MMPs in saliva can be affected by systemic and environmental conditions, such as smoking. Smoking impairs the salivary levels of cytokines and enzymes and results in lower MMP-8 and MMP-8/TIMP-1 molar ratio levels in comparison with nonsmoker subjects. ${ }^{31,46}$ A lower MMP-8 proteolytic activity has also been found in smokers in relation to ex-smoker subjects. ${ }^{53}$ These findings are similar to those found in other systemic conditions, such as pregnancy, where MMP-8 shows smaller postpartum than prepartum levels, which inversely changes in relation to pregnancy gingivitis. ${ }^{54}$ Elevated salivary MMP-8 activation has also been associated with acute myocardial infarction. ${ }^{55}$

However, some inconsistencies in the literature have been found between single salivary biomarkers, partially explained on the basis of different study populations, analytical methods, systemic illnesses, and the large variety of biomarkers involved in periodontal disease..$^{29,56-58}$ To overcome those difficulties, the cumulative risk score index considers a set of biomarkers that include $P$. gingivalis representing infection, IL-1 $\beta$ representing inflammation, and MMP-8 reflecting tissue degradation. The calculation of subscores, considering the tertile values of each biomarker, classifies the subjects into three levels of risk (I, II, and III), from the lowest to the highest one. ${ }^{29,58}$ According to recent studies, the cumulative risk score is more accurate than any of the biomarkers alone and is able to differentiate periodontitis subjects from their controls, regardless of the coronary artery disease of the patients. ${ }^{10,58}$ Recent studies have repeatedly demonstrated that commercially available 
mouthrinse aMMP-8 chair-side/point-of-care (PoC) lateral flow immunoassay (PerioSafe ${ }^{\circledR}$ and Oral Risk Indicator ${ }^{\circledR}$, Dentognostics GmHb, Jena, Germany) detects at least two sites with pathological deepened periodontal pockets and BOP but exerts low sensitivity for single deepened pockets and BOP (Figure 1). ${ }^{29,59}$ This mouthrinse aMMP-8 PoC/chairside assay is sensitive and specific enough to detect initial or early signs of periodontitis in adolescents (Figure 1). ${ }^{60}$ The cut off value of aMMP-8 lateral flow mouthrinse immune assay is $25 \mathrm{ng} / \mathrm{mL} .{ }^{60}$ The PoC aMMP-8 immunoassay PerioSafe showed $>70 \%$ sensitivity and $96 \%$ specificity. ${ }^{29,60}$

\section{MMPs as diagnostic biomarkers of periodontal/peri-implant diseases in GCF and PISF}

GCF and PISF are serum transudates that bathe the gingival sulcus in physiologic conditions. In the presence of inflammation, it becomes an exudate that carries molecules from both interstitial periodontal/peri-implant tissues and general circulation. ${ }^{61}$ Thus, GCF constituents as candidate biomarkers might reflect local periodontal and systemic inflammation, respectively. ${ }^{62}$ Higher GCF and PISF collagenolytic MMP levels, particularly of MMP-8, have been widely reported in periodontitis compared with healthy sites overcoming the protective shield provided by TIMP-1. It also associates with the levels of type I collagen degradation products, whereas the nonsurgical periodontal treatment improves clinical parameters along with the reductions of MMP-8, along with MMP-9, and -13 levels and activities. ${ }^{29,30,34,63-66}$

Currently, the challenge in biomarker site-specific GCF and PISF assessments point out a need to identify a diagnostic

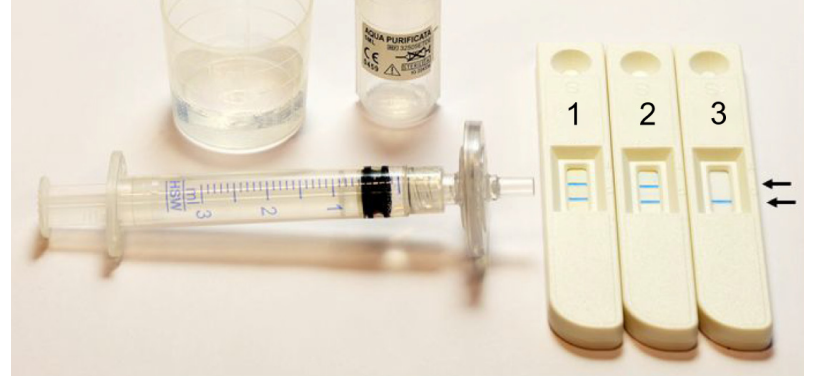

Figure I Mouthrinse aMMP-8 point-of-care/chair-side assay.

Notes: Constituents (purified rinse water, cup, and syringe with filter) and lateral flow test cassette of aMMP-8 chair-side/point-of-care oral fluid immunoassay. Two lines (indicated by arrows) indicate increased, $>25 \mathrm{mg} / \mathrm{mL}$, MMP- 8 in mouthrinse and elevated risk for periodontitis; even a thin second line indicate the risk $(I, 2)$. One line indicates no risk (3) for periodontitis, the patient is under control, patient's treatment and medication have been successful and/or is in good maintenance.

Abbreviations: MMP, matrix metalloproteinase; aMMP, active matrix metalloproteinase. array of biomarkers to account for a disease's biological profile and disclose current or future periodontal and peri-implant loss. ${ }^{6,12,67}$ Until now, few studies have evaluated biomarkers' site-specific diagnostic potential to differentiate periodontitis sites from inflamed sites with no attachment loss, as well as their predictive/prognostic potential to identify sites at risk of progression and to evaluate treatment response. ${ }^{29}$

Regarding the diagnostic potential, elevated MMP-8 levels in GCF and PISF measured by two different quantitative methods (IFMA and commercial ELISA) along with myeloperoxidase (MPO), discriminate chronic periodontitis from gingivitis and healthy sites. ${ }^{68} \mathrm{MPO}$ is an antimicrobial neutrophil-derived enzyme that catalyzes the synthesis of hypochlorous acid, reported to oxidatively activate latent proMMP-8 and -9 in vitro. ${ }^{69,70}$ In this context, gingivitis/ peri-implant mucositis represents a control for inflamed sites with no periodontal/peri-implant attachment loss. ${ }^{29}$ Another previous study incorporated gingivitis controls, testing a quantitative MMP-8 assay (IFMA) and a qualitative test-stick with similar results. ${ }^{66}$ Interestingly, there is good agreement between the different tests for GCF MMP- 8 assessments, but thresholds/cut-off values for diagnosis depend on the utilized specific monoclonal/polyclonal antibodies and detection method. ${ }^{14,66}$ Nevertheless, the findings of Mäntylä et al, ${ }^{66}$ utilizing monoclonal anti-MMP- 8 antibodies, have been confirmed by Akbari et al, ${ }^{71}$ utilizing polyclonal antiMMP-8 antibodies. Mäntylä et $\mathrm{al}^{66}$ and Akbari et $\mathrm{al}^{71}$ have independently demonstrated, thus confirming each other, that the specificity and sensitivity obtained with monoclonal and polyclonal anti-MMP-8 antibodies are practically same, validating the dip-stick strip MMP-8 chair-side GCF test kit. ${ }^{71}$ Furthermore, MMP-8 and myeloperoxidase (MPO) show a high potential for site-specific diagnosis of periodontitis determined by receiver operating characteristic curves (diagnostic accuracy 0.90-0.98). Both the markers (MMP-8 and MPO) and MMP-8 quantitative methods (IFMA and ELISA) showed very high diagnostic sensitivity and specificity discriminating from healthy and gingivitis sites, supporting their potential usefulness for $\mathrm{PoC}$ diagnostics. These results emphasize that high levels of MMP-8, along with MPO, reflect the loss of periodontal supporting tissues rather than solely inflammation. ${ }^{68}$

Significant positive correlations between collagenases (MMP-8, -13, and -14) are hinted in gingivitis and are clearly evidenced in periodontitis sites. ${ }^{68,72}$ Noteworthy, total MMP-8 levels and its active isoforms repeatedly correlate with MPO levels in periodontitis sites, suggesting that pro-MMP-8 activation by MPO-derived Hypochlorous acid represents a key activating mechanism for triggered neutrophils. 
A similar correlation is also described for MPO and MMP9. ${ }^{65}$ Altogether, clinical evidence suggests that inflammation contributes to the loss of periodontal homeostasis through both proteolytic and oxidative mechanisms activating key MMPs in periodontal tissues. ${ }^{69,72}$

In a recent study, the site-specific diagnostic accuracy of MMP-8 and MPO in GCF was further confirmed in chronic periodontitis and novel biomarker candidates emerged, including MMP-9 and soluble tartrate-resistant acid phosphatase (TRAP)-5; the latter is regarded as a direct marker of osteoclastic activity and bone resorption. ${ }^{73}$ Moreover, the potential of GCF side-diagnosis in apical periodontitis was explored. The performance of MMP-8, MPO, MMP-9, and TRAP-5 was not limited to their high diagnostic accuracy in chronic periodontitis (0.91-1.0), but they also reflected disease severity, correlating to $\mathrm{PD}, \mathrm{CAL}$, and/or radiographic bone level. Moreover, data from our group (Hernández-Ríos $\mathrm{P}$ and Hernández M) demonstrate a positive correlation between bone resorption, reflected by TRAP, MMP-8, and MMP-9 activities (correlation coefficient $=0.81$ and 0.74 , respectively, $P<0.05)$. Although GCF volume and total protein concentration are limited $\left(<1 \mu \mathrm{L}\right.$ and $<1 \mathrm{mg} / \mathrm{mL}$, respectively), ${ }^{62}$ these enzymes, as opposed to proinflammatory and osteolytic cytokines, such as IL-1 $\beta$, IL-6, tumor necrosis factor- $\alpha$, receptor activator of nuclear factor $\mathrm{\kappa B}$ ligand, and osteoprotegerin, can be easily detected and quantified in GCF, representing high diagnostic accuracy biomarkers for site-specific diagnosis of chronic periodontitis. Moreover, proteolytic metalloproteinases can regulate osteolytic cytokines, such as IL-6 and receptor activator of nuclear factor $\kappa \mathrm{B}$ ligand. ${ }^{74-76}$

The changes in GCF composition do not seem to be limited to chronic periodontitis. MMP-8 and -9 can also differentiate between healthy and asymptomatic apical periodontitis (AAP); at higher levels, they discriminate chronic periodontitis from AAP and show diagnostic potential for the later, though lower compared with chronic periodontitis (accuracy of 0.90-0.93 for active MMP-9 and -8 ELISA, respectively). ${ }^{5}$ The hallmark of AAP is the destruction of periradicular periodontal tissues leading to the formation of an osteolytic apical lesion in the absence of clinical symptoms. ${ }^{2}$ Few earlier studies including GCF analysis have been conducted in AAP, but the results are consistent with these findings. Higher levels of pro-MMP-9 were reported for the first time in teeth with apical lesions and healthy contra lateral controls in a split mouth design. ${ }^{77}$ Accordingly, higher gelatinolytic MMP-9 activity in human apical lesions was later confirmed in comparison with healthy periodontal ligament controls. ${ }^{8}$ In addition to MMP-9, MMP-8 has also been immunolocalized to apical lesions and inflamed pulp tissue and its levels decreased with statistical significance after intracanal calcium hydroxide medication $;{ }^{78}$ nevertheless, no previous studies have been conducted in GCF.

The mechanisms explaining how GCF composition might be modified during AAP remain unknown. Bacteria, their products, and/or inflammatory mediators may diffuse from the apical lesions and/or infected endodontic canals either through periodontal circulation or periodontal ligament through root fractures, microcracks, exposed dentinal tubules, and lateral canals. Inflammatory mediators might also extravasate from systemic circulation. Accordingly, recent studies support that AP might associate with lowgrade systemic inflammation. ${ }^{79}$ Overall, destructive chronic inflammatory processes of the periodontium at both marginal and periapical levels can modify GCF composition with the potential for periodontal-side diagnostics and eventually to assess systemic involvement. ${ }^{80}$

Emerging evidence supports that host-derived MMPs can also be applied to evaluate the risk and course of periodontitis progression. MMP-8 levels and MMP-13 activity were higher in nontreated progressing versus nonprogressing periodontitis sites, categorized by the tolerance method, although they are statistically significant only for MMP-13. ${ }^{72,81}$ After nonsurgical periodontal treatment, MMP-8 along with MPO levels were reduced, but the association of MPO and MMP-8 persisted in progressive sites. ${ }^{72}$ In another study, stable sites that maintained their CAL and PD improvements after periodontal treatment up to the end of the maintenance phase (1 year follow-up) showed a sustained reduction of MMP-8 levels measured by IFMA and ELISA; whereas unstable sites, that showed further CAL and PD positive changes, showed marked MMP-8 picks during the maintenance phase. ${ }^{14}$ In line with these findings, elevated concomitant levels of IL-1 $\beta$ and MMP-8 after nonsurgical periodontal treatment (1 year) associated with increased risk of subsequent periodontal attachment loss in postmenopausal women ( 2 years), identifying sites more prone to progress. Adjunctive subantimicrobial dose doxycycline medication was effective to drop the risk of periodontitis progression along with MMP-8 levels. ${ }^{82}$

The ability of a panel including host-derived and bacterial biomarkers in GCF saliva, serum, and dental plaque is assessed in progressing and stable chronic periodontitis patients. Progression is defined as any site undergoing CAL $>2 \mathrm{~mm}$ from the baseline measurement, followed by 6 months untreated (disease monitoring phase) and 6 months after nonsurgical treatment (recovery phase). In GCF, mild and moderate periodontitis groups showed significantly reduced MMP-8 levels during recovery. Statistically significant higher baseline levels are found for IL-1 $\beta$, osteoprotegerin, MMP-8, 
and -9 in progressive versus stable sites. The authors state that singular analysis of GCF biomarkers is useful to predict periodontal disease progression, but when combined with clinical and other biological measures, the predictability rises. The greatest degree of sensitivity for progression is found for saliva biomarkers and the greatest specificity for GCF. Serum, on the other hand, shows no prognostic role to identify future periodontal progression. ${ }^{13}$

It is noteworthy that tobacco smoking can influence MMP-8 levels in GCF from periodontitis subjects. Tobacco seems to downregulate MMP-8 levels; however, the levels remain high after nonsurgical treatment in progressive sites of both nonsmokers and smokers. ${ }^{32}$ Moreover, high MMP-8 levels at baseline significantly predict a weak treatment response in smokers, defined by the patterns of CAL reductions. According to their findings, the authors recommend monitoring of MMP-8 levels during the maintenance period. ${ }^{83}$ For this purpose, a PoC/chair-side oral fluid MMP-8 assay (PerioSafe) is a very practical, inexpensive, and fast diagnostic tool to be performed by dental and medical professionals, and even by the patients themselves (Figure 1). Overall, testing of MMP-8 in GCF, PISF, and/or oral fluids (saliva and mouthrinse) represents a promising chair-side/PoC diagnostic tool to identify patients' and sites at risk for periodontal/peri-implant disease progression and predict treatment response.

\section{Acknowledgments}

The studies in our laboratories have been supported by grants from the National Fund for Scientific and Technological Development from Chilean Government (FONDECYT) 1160741, 1120138, and 1090461; the Helsinki University Central Hospital Research Foundation, Helsinki, Finland; and Karolinska Institutet, Stockholm, Sweden.

\section{Disclosure}

Professor Timo Sorsa is an inventor of US patents 5652227 , 5736341,5866432 , and 6143476 . The authors report no other conflicts of interest in this work.

\section{References}

1. Savage A, Eaton KA, Moles DR, Needleman I. A systematic review of definitions of periodontitis and methods that have been used to identify this disease. J Clin Periodontol. 2009;36(6):458-467.

2. Garrido M, Dezerega A, Castro-Martínez A, Hernández M. Host-derived biomarkers in gingival crevicular fluid for complementary diagnosis of apical periodontitis. World J Stomatol. 2014;3(2):19-24.

3. Hernández P, Mäntylä P, Tervahartiala T, Sorsa T, Hernández M. Oralfluid MMP analysis in the complementary diagnosis of periodontal diseases. Rev Clin Periodoncia Implantol Rehabil Oral. 2012;5(3): $150-153$
4. Hernández M, Vernal R, Mäntyla P, Tervahartiala T, Sorsa T, Gamonal J. Chronic periodontitis: the role of immuno-inflammatory response in the pathogenesis of chronic periodontitis and development of chairside point of care diagnostics in periodontitis and related systemic inflammation. In: Buduneli N, editor. Pathogenesis and Treatment of Periodontitis. Rijeka: InTech; 2012:33-54.

5. Baeza M, Garrido M, Hernández-Ríos P, et al. Diagnostic accuracy for apical and chronic periodontitis biomarkers in gingival crevicular fluid: an exploratory study. J Clin Periodontol. 2016;43(1):34-45.

6. Buduneli N, Kinane DF. Host-derived diagnostic markers related to soft tissue destruction and bone degradation in periodontitis. $J$ Clin Periodontol. 2011;38(Suppl 11):85-105.

7. Pussinen PJ, Paju S, Mäntylä P, Sorsa T. Serum microbial- and hostderived markers of periodontal diseases: a review. Curr Med Chem. 2007;14(22):2402-2412.

8. Dezerega A, Madrid S, Mundi V, et al. Pro-oxidant status and matrix metalloproteinases in apical lesions and gingival crevicular fluid as potential biomarkers for asymptomatic apical periodontitis and endodontic treatment response. J Inflamm (Lond). 2012;9(1):8.

9. American Academy of Periodontology. Comprehensive periodontal therapy: a statement by the American Academy of Periodontology*. J Periodontol. 2011;82(7):943-949.

10. Salminen A, Gursoy UK, Paju S, et al. Salivary biomarkers of bacterial burden, inflammatory response, and tissue destruction in periodontitis. J Clin Periodontol. 2014;41(5):442-450.

11. Armitage GC. The complete periodontal examination. Periodontol 2000. 2004;34:22-33.

12. Offenbacher S, Barros SP, Singer RE, Moss K, Williams RC, Beck JD. Periodontal disease at the biofilm-gingival interface. J Periodontol. 2007;78(10):1911-1925.

13. Kinney JS, Morelli T, Oh M, et al. Crevicular fluid biomarkers and periodontal disease progression. J Clin Periodontol. 2014;41(2):113-120.

14. Sorsa T, Hernandez M, Leppilahti J, Munjal S, Netuschil L, Mäntylä P. Detection of gingival crevicular fluid MMP-8 levels with different laboratory and chair-side methods. Oral Dis. 2010;16(1):39-45.

15. Sahingur SE, Cohen RE. Analysis of host responses and risk for disease progression. Periodontol 2000. 2004;34:57-83.

16. Sorsa T, Tjaderhane L, Salo T. Matrix metalloproteinases (MMPs) in oral diseases. Oral Dis. 2004;10(6):311-318.

17. Uitto VJ, Tryggvason K, Sorsa T. Collagenolytic enzymes in periodontal diseases. Proc Finn Dent Soc. 1987;83(3):119-130.

18. Verma RP, Hansch C. Matrix metalloproteinases (MMPs): chemical-biological functions and (Q)SARs. Bioorg Med Chem. 2007;15(6):2223-2268.

19. Folgueras AR, Pendás AM, Sánchez LM, López-Otín C. Matrix metalloproteinases in cancer: from new functions to improved inhibition strategies. Int J Dev Biol. 2004;48(5-6):411-424.

20. McQuibban GA, Butler GS, Gong JH, et al. Matrix metalloproteinase activity inactivates the CXC chemokine stromal cell-derived factor-1. J Biol Chem. 2001;276(47):43503-43508.

21. McQuibban GA, Gong JH, Wong JP, Wallace JL, Clark-Lewis I, Overall CM. Matrix metalloproteinase processing of monocyte chemoattractant proteins generates CC chemokine receptor antagonists with anti-inflammatory properties in vivo. Blood. 2002;100(4): $1160-1167$.

22. Butler GS, Overall CM. Matrix metalloproteinase processing of signaling molecules to regulate inflammation. Periodontol 2000. 2013;63(1):123-148.

23. Kuula H, Salo T, Pirilä E, et al. Local and systemic responses in matrix metalloproteinase 8-deficient mice during Porphyromonas gingivalisinduced periodontitis. Infect Immun. 2009;77(2):850-859.

24. Hernández M, Gamonal J, Salo T, et al. Reduced expression of lipopolysaccharide-induced CXC chemokine in Porphyromonas gingivalis-induced experimental periodontitis in matrix metalloproteinase-8 null mice. J Periodontal Res. 2011;46(1):58-66. 
25. Kessenbrock K, Plaks V, Werb Z. Matrix metalloproteinases: regulators of the tumor microenvironment. Cell. 2010;141(1): 52-67.

26. Sorsa T, Mäntylä P, Tervahartiala T, Pussinen PJ, Gamonal J, Hernandez M. MMP activation in diagnostics of periodontitis and systemic inflammation. J Clin Periodontol. 2011;38(9):817-819.

27. Sorsa T, Tjäderhane L, Konttinen YT, et al. Matrix metalloproteinases: contribution to pathogenesis, diagnosis and treatment of periodontal inflammation. Ann Med. 2006;38(5):306-321

28. Sbardella D, Fasciglione GF, Gioia M, et al. Human matrix metalloproteinases: an ubiquitarian class of enzymes involved in several pathological processes. Mol Aspects Med. 2012;33(2): 119-208.

29. Sorsa T, Gursoy UK, Nwhator S, et al. Analysis of matrix metalloproteinases, especially MMP-8, in gingival creviclular fluid, mouthrinse and saliva for monitoring periodontal diseases. Periodontol 2000. 2016;70(1):142-163.

30. Golub LM, Lee HM, Stoner JA, et al. Subantimicrobial-dose doxycycline modulates gingival crevicular fluid biomarkers of periodontitis in postmenopausal osteopenic women. J Periodontol. 2008;79(8):1409-1418.

31. Heikkinen AM, Sorsa T, Pitkäniemi J, et al. Smoking affects diagnostic salivary periodontal disease biomarker levels in adolescents. J Periodontol. 2010;81(9):1299-1307.

32. Mäntylä P, Stenman M, Kinane DF, et al. Monitoring periodontal disease status in smokers and nonsmokers using a gingival crevicular fluid matrix metalloproteinase-8-specific chair-side test. J Periodontal Res. 2006;41(6):503-512.

33. Tervahartiala T, Pirilä E, Ceponis A, et al. The in vivo expression of the collagenolytic matrix metalloproteinases (MMP-2, -8, -13, and -14) and matrilysin (MMP-7) in adult and localized juvenile periodontitis. J Dent Res. 2000;79(12):1969-1977.

34. Hernandez M, Valenzuela MA, Lopez-Otin C, et al. Matrix metalloproteinase-13 is highly expressed in destructive periodontal disease activity. J Periodontol. 2006;77(11):1863-1870.

35. Holliday LS, Welgus HG, Fliszar CJ, Veith GM, Jeffrey JJ, Gluck SL. Initiation of osteoclast bone resorption by interstitial collagenase. J Biol Chem. 1997;272(35):22053-22058.

36. Rydziel S, Durant D, Canalis E. Platelet-derived growth factor induces collagenase 3 transcription in osteoblasts through the activator protein 1 complex. J Cell Physiol. 2000;184(3):326-333.

37. Hill PA, Docherty AJ, Bottomley KM, et al. Inhibition of bone resorption in vitro by selective inhibitors of gelatinase and collagenase. Biochem J. 1995;308(Pt 1):167-175.

38. Mäkelä M, Salo T, Uitto VJ, Larjava H. Matrix metalloproteinases (MMP-2 and MMP-9) of the oral cavity: cellular origin and relationship to periodontal status. J Dent Res. 1994;73(8):1397-1406.

39. Navazesh M, Kumar SK. Measuring salivary flow: challenges and opportunities. J Am Dent Assoc. 2008;139(Suppl):35S-40S.

40. Kaufman E, Lamster IB. The diagnostic applications of saliva - a review. Crit Rev Oral Biol Med. 2002;13(2):197-212.

41. Giannobile WV, Beikler T, Kinney JS, Ramseier CA, Morelli T, Wong DT. Saliva as a diagnostic tool for periodontal disease: current state and future directions. Periodontol 2000. 2009;50:52-64.

42. Miller CS, Foley JD, Bailey AL, et al. Current developments in salivary diagnostics. Biomark Med. 2010;4(1):171-189.

43. Al-Tarawneh SK, Border MB, Dibble CF, Bencharit S. Defining salivary biomarkers using mass spectrometry-based proteomics: a systematic review. OMICS. 2011;15(6):353-361.

44. Furuholm J, Sorsa T, Qvarnström M, et al. Salivary matrix metalloproteinase- 8 in patients with and without coronary heart disease may indicate an increased susceptibility to periodontal disease. J Periodontal Res. 2006;41(5):486-489.

45. Costa PP, Trevisan GL, Macedo GO, et al. Salivary interleukin-6, matrix metalloproteinase- 8 , and osteoprotegerin in patients with periodontitis and diabetes. J Periodontol. 2010;81(3):384-391.
46. Rathnayake N, Akerman S, Klinge B, et al. Salivary biomarkers of oral health: a cross-sectional study. J Clin Periodontol. 2013;40(2):140-147.

47. Miller CS, King CP Jr, Langub MC, Kryscio RJ, Thomas MV. Salivary biomarkers of existing periodontal disease: a cross-sectional study. J Am Dent Assoc. 2006;137(3):322-329.

48. Karaman A, Torun E, Gursoy S, Yurci A, Ozbakir O. Efficacy of Ankaferd Blood Stopper in postpolypectomy bleeding. JAltern Complement Med. 2010;16(10):1027-1028

49. Gursoy UK, Kononen E, Huumonen S, et al. Salivary type I collagen degradation end-products and related matrix metalloproteinases in periodontitis. J Clin Periodontol. 2013;40(1):18-25.

50. Ramseier CA, Kinney JS, Herr AE, et al. Identification of pathogen and host-response markers correlated with periodontal disease. J Periodontol. 2009;80(3):436-446.

51. Uitto VJ, Suomalainen K, Sorsa T. Salivary collagenase. Origin, characteristics and relationship to periodontal health. $J$ Periodontal Res. 1990;25(3):135-142.

52. Sexton WM, Lin Y, Kryscio RJ, Dawson DR 3rd, Ebersole JL, Miller CS. Salivary biomarkers of periodontal disease in response to treatment. J Clin Periodontol. 2011;38(5):434-441.

53. Liede KE, Haukka JK, Hietanen JH, Mattila MH, Rönkä H, Sorsa T. The association between smoking cessation and periodontal status and salivary proteinase levels. J Periodontol. 1999;70(11): 1361-1368.

54. Gursoy M, Kononen E, Tervahartiala T, Gursoy UK, Pajukanta R, Sorsa T. Longitudinal study of salivary proteinases during pregnancy and postpartum. J Periodontal Res. 2010;45(4):496-503.

55. Buduneli E, Mäntylä P, Emingil G, et al. Acute myocardial infarction is reflected in salivary matrix metalloproteinase- 8 activation level. J Periodontol. 2011;82(5):716-725.

56. Mirrielees J, Crofford LJ, Lin Y, et al. Rheumatoid arthritis and salivary biomarkers of periodontal disease. J Clin Periodontol. 2010;37(12):1068-1074.

57. Palm F, Lahdentausta L, Sorsa T, et al. Biomarkers of periodontitis and inflammation in ischemic stroke: a case-control study. Innate Immun. 2014;20(5):511-518.

58. Gursoy UK, Könönen E, Pussinen PJ, et al. Use of host- and bacteriaderived salivary markers in detection of periodontitis: a cumulative approach. Dis Markers. 2011;30(6):299-305.

59. Nwhator SO, Ayanbadejo PO, Umeizudike KA, et al. Clinical correlates of a lateral-flow immunoassay oral risk indicator. $J$ Periodontol. 2014;85(1):188-194.

60. Heikkinen AM, Nwhator SO, Rathnayake N, Mäntylä P, Vatanen P, Sorsa T. Pilot study on oral health status as assessed by an active matrix metalloproteinase- 8 chairside mouthrinse test in adolescents. J Periodontol. 2016;87(1):36-40.

61. Griffiths GS. Formation, collection and significance of gingival crevice fluid. Periodontol 2000. 2003;31:32-42.

62. Hernández M, Martinez B, Tejerina JM, Valenzuela MA, Gamonal J. MMP-13 and TIMP-1 determinations in progressive chronic periodontitis. J Clin Periodontol. 2007;34(9):729-735.

63. Pozo P, Valenzuela MA, Melej C, et al. Longitudinal analysis of metalloproteinases, tissue inhibitors of metalloproteinases and clinical parameters in gingival crevicular fluid from periodontitis-affected patients. J Periodontal Res. 2005;40(3):199-207.

64. Golub LM, Lee HM, Greenwald RA, et al. A matrix metalloproteinase inhibitor reduces bone-type collagen degradation fragments and specific collagenases in gingival crevicular fluid during adult periodontitis. Inflamm Res. 1997;46(8):310-319.

65. Marcaccini AM, Meschiari CA, Zuardi LR, et al. Gingival crevicular fluid levels of MMP-8, MMP-9, TIMP-2, and MPO decrease after periodontal therapy. J Clin Periodontol. 2010;37(2):180-190.

66. Mäntylä P, Stenman M, Kinane DF, et al. Gingival crevicular fluid collagenase-2 (MMP-8) test stick for chair-side monitoring of periodontitis. J Periodontal Res. 2003;38(4):436-439. 
67. Kinane DF, Preshaw PM, Loos BG; Working Group 2 of Seventh European Workshop on Periodontology. Host-response: understanding the cellular and molecular mechanisms of host-microbial interactions - consensus of the Seventh European Workshop on Periodontology. J Clin Periodontol. 2011;38(Suppl 11):44-48.

68. Leppilahti JM, Hernández-Ríos PA, Gamonal JA, et al. Matrix metalloproteinases and myeloperoxidase in gingival crevicular fluid provide site-specific diagnostic value for chronic periodontitis. J Clin Periodontol. 2014;41(4):348-356.

69. Saari H, Suomalainen K, Lindy O, Konttinen YT, Sorsa T. Activation of latent human neutrophil collagenase by reactive oxygen species and serine proteases. Biochem Biophys Res Commun. 1990;171(3):979-987.

70. Spallarossa P, Garibaldi S, Barisione C, et al. Postprandial serum induces apoptosis in endothelial cells: role of polymorphonuclear-derived myeloperoxidase and metalloproteinase-9 activity. Atherosclerosis. 2008;198(2):458-467.

71. Akbari G, Prabhuji ML, Karthikeyan BV, Raghunatha K, Narayanan R. Analysis of matrix metalloproteinase-8 levels in gingival crevicular fluid and whole mouth fluid among smokers and nonsmokers using enzyme-linked immune-sorbent assay and a novel chair-side test. J Indian Soc Periodontol. 2015;19(5):525-530.

72. Hernández M, Gamonal J, Tervahartiala T, et al. Associations between matrix metalloproteinase- 8 and -14 and myeloperoxidase in gingival crevicular fluid from subjects with progressive chronic periodontitis: a longitudinal study. J Periodontol. 2010;81(11):1644-1652.

73. Mose S, Menzel C, Kurth AA, et al. Tartrate-resistant acid phosphatase $5 \mathrm{~b}$ as serum marker of bone metabolism in cancer patients. Anticancer Res. 2003;23(3C):2783-2788.

74. Bostanci N, Emingil G, Afacan B, et al. Tumor necrosis factor-alphaconverting enzyme (TACE) levels in periodontal diseases. J Dent Res. 2008;87(3):273-277.

75. Cavalla F, Osorio C, Paredes R, et al. Matrix metalloproteinases regulate extracellular levels of SDF-1/CXCL12, IL-6 and VEGF in hydrogen peroxide-stimulated human periodontal ligament fibroblasts. Cytokine. 2015;73(1):114-121.
76. Nannuru KC, Futakuchi M, Varney ML, Vincent TM, Marcusson EG, Singh RK. Matrix metalloproteinase (MMP)-13 regulates mammary tumor-induced osteolysis by activating MMP9 and transforming growth factor-beta signaling at the tumor-bone interface. Cancer Res. 2010;70(9):3494-3504.

77. Belmar MJ, Pabst C, Martinez B, Hernández M. Gelatinolytic activity in gingival crevicular fluid from teeth with periapical lesions. Oral Surg Oral Med Oral Pathol Oral Radiol Endod. 2008;105(6):801-806.

78. Wahlgren J, Salo T, Teronen O, Luoto H, Sorsa T, Tjaderhane L. Matrix metalloproteinase-8 (MMP-8) in pulpal and periapical inflammation and periapical root-canal exudates. Int Endod J. 2002;35(11): 897-904.

79. Gomes MS, Blattner TC, Sant'Ana Filho M, et al. Can apical periodontitis modify systemic levels of inflammatory markers? A systematic review and meta-analysis. J Endod. 2013;39(10):1205-1217.

80. Sorsa T, Tervahartiala T, Leppilahti J, et al. Collagenase-2 (MMP-8) as a point-of-care biomarker in periodontitis and cardiovascular diseases. Therapeutic response to non-antimicrobial properties of tetracyclines. Pharmacol Res. 2011;63(2):108-113.

81. Hernández Ríos M, Sorsa T, Obregón F, et al. Proteolytic roles of matrix metalloproteinase (MMP)-13 during progression of chronic periodontitis: initial evidence for MMP-13/MMP-9 activation cascade. J Clin Periodontol. 2009;36(12):1011-1017.

82. Reinhardt RA, Stoner JA, Golub LM, et al. Association of gingival crevicular fluid biomarkers during periodontal maintenance with subsequent progressive periodontitis. J Periodontol. 2010;81(2):251-259.

83. Leppilahti JM, Kallio MA, Tervahartiala T, Sorsa T, Mäntylä P. Gingival crevicular fluid matrix metalloproteinase- 8 levels predict treatment outcome among smokers with chronic periodontitis. J Periodontol. 2014;85(2):250-260.
Metalloproteinases In Medicine

\section{Publish your work in this journal}

Metalloproteinases In Medicine is an international, peer reviewed, open access journal that aims to provide a platform for the discussion and dissemination of knowledge about the role that metalloproteinases - such as matrix metalloproteinases (MMP), ADAMs, ADAMTSs, and astacins, as well as their inhibitors - play in diseases.

\section{Dovepress}

The manuscript management system is completely online and includes a very quick and fair peer review system, which is all easy to use. Visit http://www.dovepress.com/testimonials.php to read real quotes from published authors. 